\title{
Spectrum of Imaging Findings In Rotator Cuff Tears - A Descriptive Review
}

\section{Abstract}

\author{
Anupama Patil $1^{1}$, Joban Babhulkar ${ }^{1}$, Pranav Mahadeokar ${ }^{1}$
}

The rotator cuff is instrumental in movements of the shoulder, while at the same time producing balanced compressive forces to stabilise the glenohumeral joint (dynamic stabiliser) Cuff tears are usually diagnosed clinically but before a decision regarding surgery is taken, some form of cross-sectional imaging, USG and/or MRI, is a must. This descriptive review is an attempt to highlight key concepts from the existing literature, along with our experience to image these injuries and assist the clinicians to formulate protocols in the day to day management of rotator cuff pathologies.

Keywords: Rotator cuffinjuries; Cuff tears; Cuffarthropathy; Post operative imaging.

\section{Introduction}

Rotator cuff injury chances increases with age, with approximately $25 \%$ people over the age of 60 and $50 \%$ of individuals over 80 experiencing some sort of rotator cuff injury [1].

The rotator cuff is instrumental in movements of the shoulder, while at the same time producing balanced compressive forces to stabilise the glenohumeral joint (dynamic stabiliser).

Clinically, weakness or impingement along with "arc of pain" sign is present (pain while lowering the abducted arm [2] .

Cuff tears are usually diagnosed clinically but before a decision regarding surgery is taken, some form of cross-sectional imaging, USG and /or MRI, is a must. In the order of affection, supraspinatus is most commonly involved, followed by infraspinatus, subscapularis and teres minor [3].

Surgical or non surgical management of cuff tears depends on integrity of rotator cuff tendons in which imaging is indispensible [4].

\author{
Aim of Imaging \\ 1-Diagnosis of rotator cuff tear \\ 2- Dimensions and exact extent of the tear : single /multiple tendons, \\ partial/full thickness \\ 3 - Degree of retraction of the torn tendon \\ 4-Shape of the tear \\ 5- Condition of the involved \\ tendon (normal/degenerated/fibrotic/calcified) \\ 6-Size of muscle belly and degree of fatty infiltration \\ 7-Condition of other structures: long head of biceps, labrum, rotator \\ interval \\ 8-Factors causing external impingement (coraco-acromial arch) \\ 9- Cuff arthropathy \\ 10- Post operative imaging
}

Before any cross-sectional imaging is obtained, radiographs of the shoulder are a must.

The primary reason to obtain a shoulder radiograph is to exclude a fracture. Additionally it can provide information about glenohumeral articulation, osseous abnormalities as well as soft tissue pathologies eg. calcific tendinopathy, etc.

\section{Various Radiographic Techniques Include:}

\section{(1)Standard AP View}

Most commonly obtained radiograph, helpful in setting of trauma for fracture evaluation, to look for calcific deposition in the tendons or bursae around the joint, look for keel spurs and in suspected cases of osteoarthritis.

\section{(2) YView}

- Useful in suspected associated shoulder dislocations and scapula fractures, and in the evaluation of degenerative disease.

- It enables us to see the acromion and the coracoid process en profile.

- The scapula is visualized in a lateral profile, giving the appearance of the 'Y'.

- Coracoid process and acromion process form the upper limbs of the 'Y'.

- The humeral head projects at the base of the ' $\mathrm{Y}$ '

\section{(3)Axial View (arm in 30 degree abduction)}

It is predominantly used to assess glenohumeral articulation in suspected associated shoulder disclocation.

\section{(4) Apical oblique view(AP image with 45 degree caudal angulation of beam) \\ Also called the Garth view of the shoulder, used to evaluate}

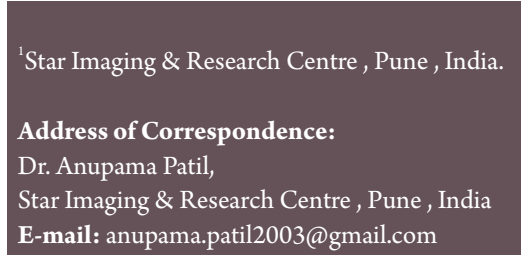

E-mail: anupama.patil2003@gmail.com

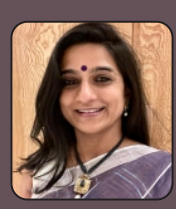

Dr. Anupama Patil

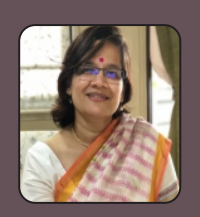

Dr. Joban Babhulkar Dr. Pranav Mahadeokar

Submitted: 18 March 2021; Reviewed: 02 April 2021; Accepted: 15 April 2021; Published: 10 June 2021

Asian Journal of Arthroscopy | ISSN 2456-1169| Available on www.asianarthroscopy.com | DOI:10.13107/aja.2021.v06i01.020|

This is an open access journal, and articles are distributed under the terms of the Creative Commons Attribution Non-Commercial-Share Alike 4.0 License (http://creativecommons.org/licenses/by-nc-sa/4.0) which allows others to remix, tweak, and build upon the work non-commercially as long as appropriate credit is given and the new creation are licensed under the identical terms. 


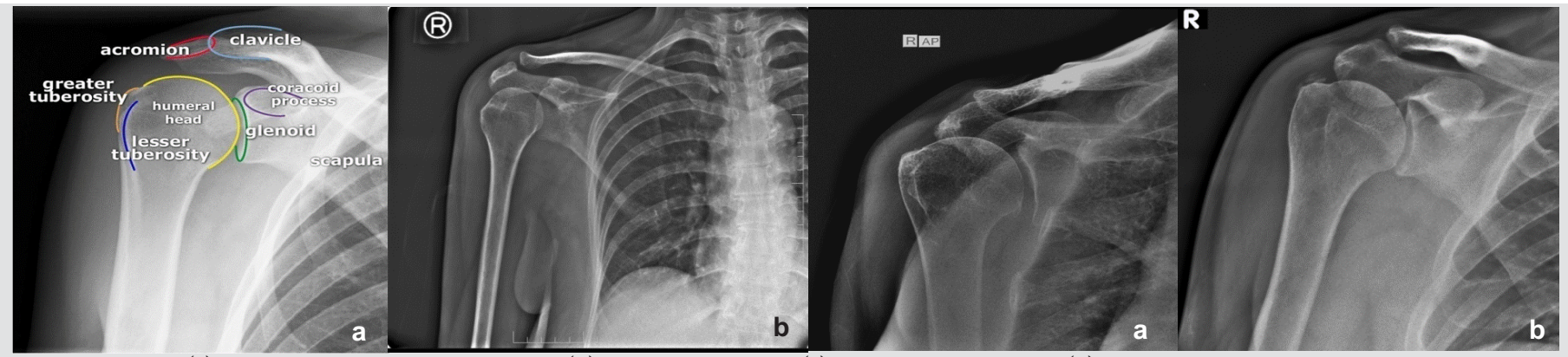

Figure 1: (a) Standard AP radiograph labelled, (b) Frontal projection, (c) Calcific tendinopathy, (d) Downsloping acromion with Keel spur

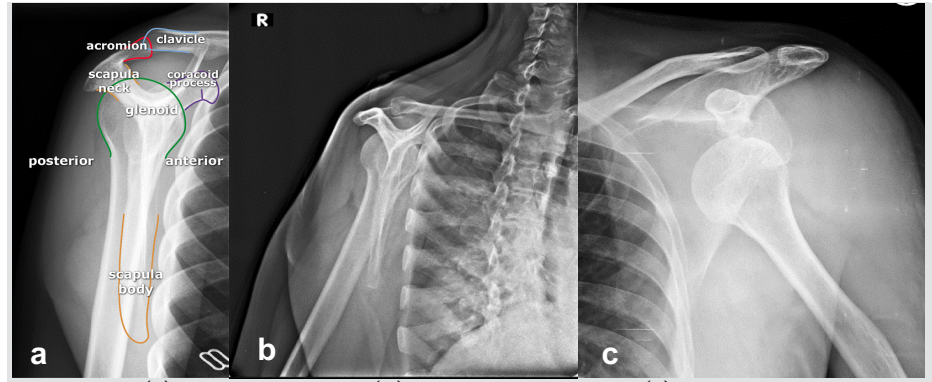

Figure 2: (a) Y view labelled, (b) Frontal projection, (c) Anterior dislocation of humeral head

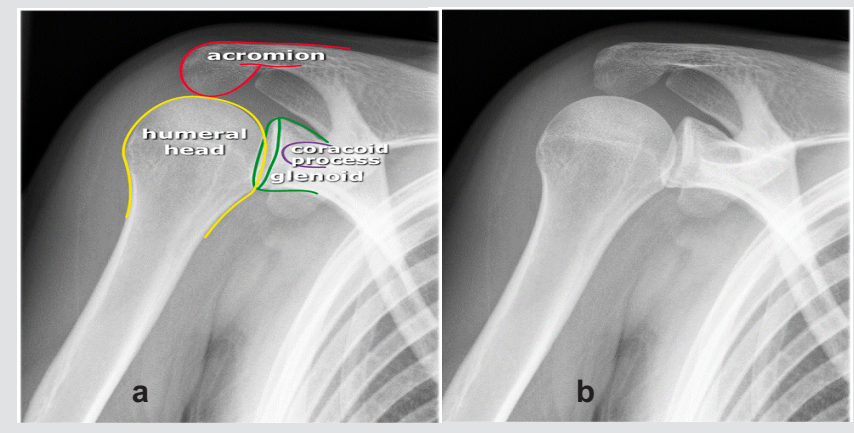

Figure 4: (a) Apical oblique view labelled (b) Actual projection

glenohumeral joint for associated dislocation and also in trauma particularly to assess the bony glenoid .

It is generally obtained as an alternative to the 'Y view' in the setting of trauma.

Bankarts and Hill Sachs lesions are well visualized in this view.

\section{(5)Stryker Notch view(AP image with 15 degree cranial} angulation of beam)

(A) Most essential in diagnosing posterior lesions of humeral head like Hill Sach lesions if associated dislocation is present.
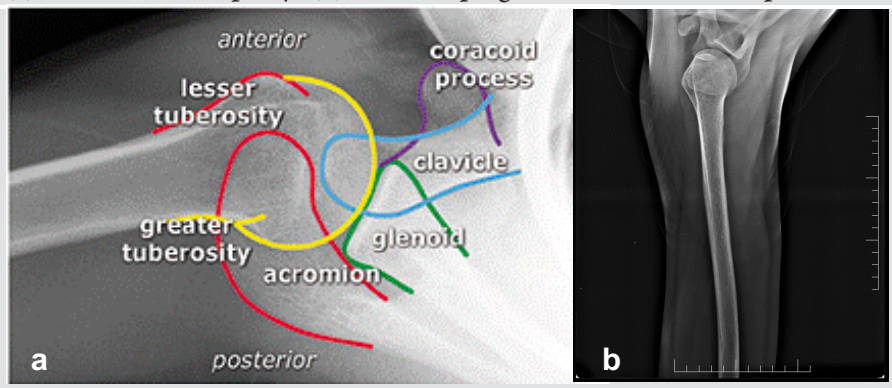

Figure 3: (a) Axial view labelled (b) Radiographic projection

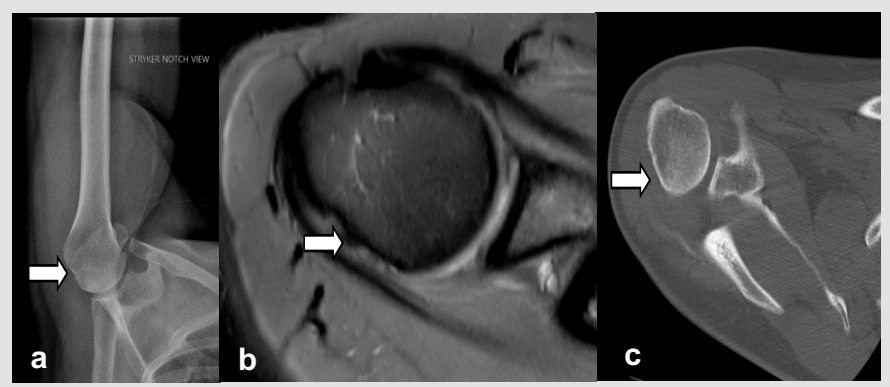

Figure 5: (a) Hill Sach's lesion demonstrated in Stryker notch projection (b) Axial PDFS MRI (c) Axial CT sequences

\section{Imaging in Cuff Tear}

USG and/or MRI are used in the definitive evaluation of cuff tears

\section{(1) Diagnosis of Cuff Tear}

Diagnosis of a cuff tear can usually be made clinically.

Imaging is however required to confirm the diagnosis of a full thickness tear and sometimes to assist in making the diagnosis of a partial thickness tear.

In patients with $\mathrm{h} / \mathrm{o}$ trauma, the greater tuberosity may look normal on the radiographs and MRI/CT is required to diagnose a fracture.
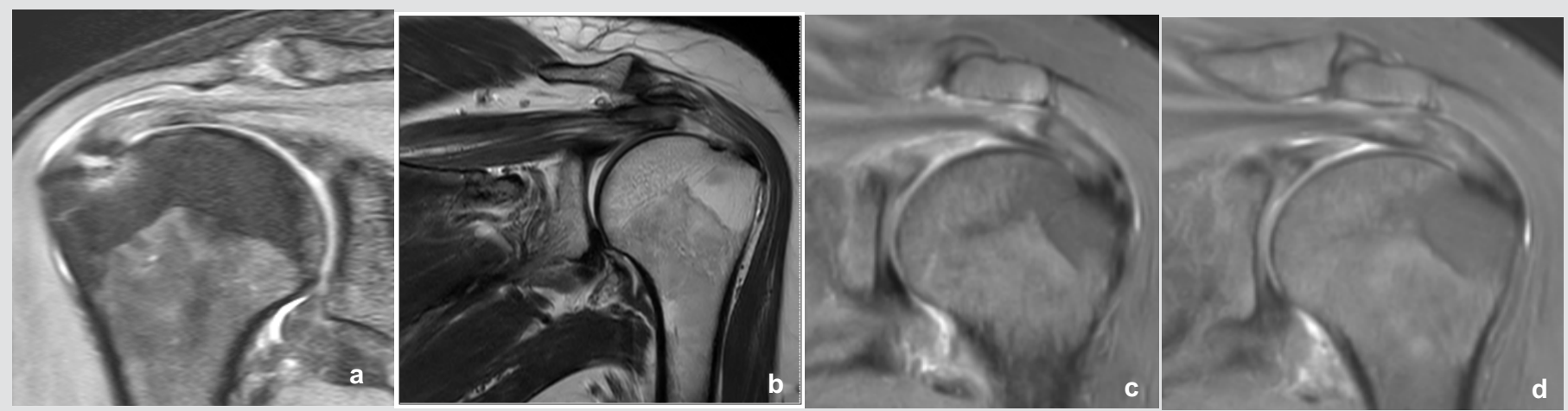

Figure 6: (a) Types of tear : insertional tear (b) Critical zone tear (c, d) Proximal/myotendinous junction tear 


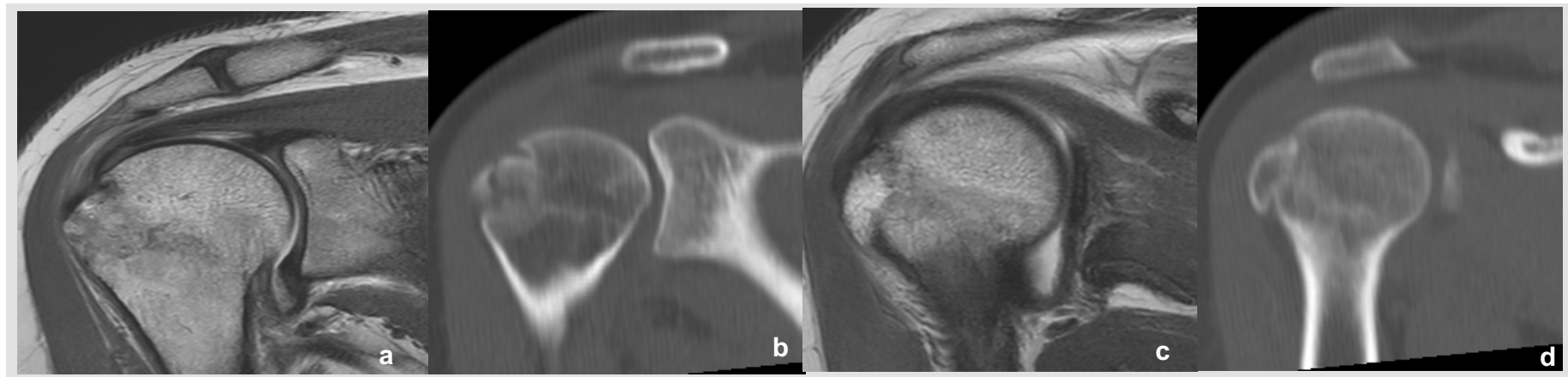

Figure 8: (a, c) Greater tuberosity fracture demonstrated on coronal PDFS MRI (c, d) Coronal CT images

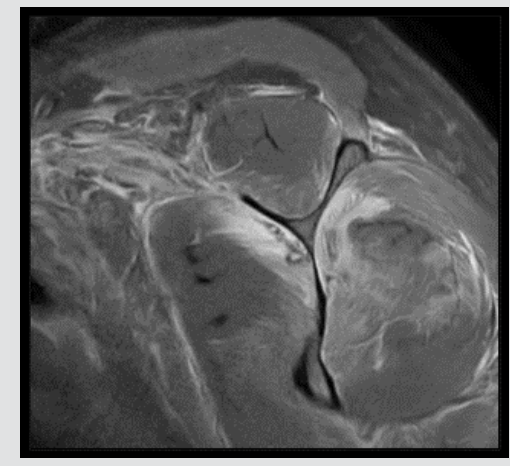

Figure 7: Muscle belly edema in the infraspinatous and teres minor with epimysial strain pattern in the supraspinatous muscle belly

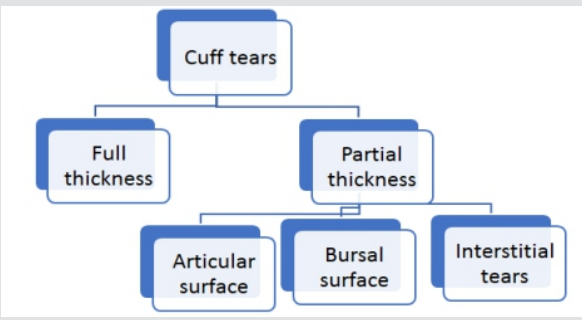

Figure 9: Classification of cuff tears

\section{(2) Dimensions of Tear}

A) Thickness of tear.

B) Antero-posterior extent of supraspinatous tear and SI extent of subscapularis/infraspinatous/teres minor tear.

C) Is the tear extending to involve adjacent tendons as well

Rotator cuff tears are well seen and easily analysed for thickness and extent on ultrasonography.

\section{USG:}

\section{Full Thickness Tear:}

The US appearance of a full thickness tear usually depends upon the amount of fluid in the subacromial bursa where the tear appears as a focal hypoechoic defect which fills up with fluid. Massive tears lead to complete non-visualisation of the cuff tendons in standard scans and the appearance of a naked greater tuberosity which is apposed directly by the overlying deltoid muscle. Indirect signs of a full thickness tear include focal herniation of the deltoid muscle and the subdeltoid fat into the focal defect, appreciated better on dynamic

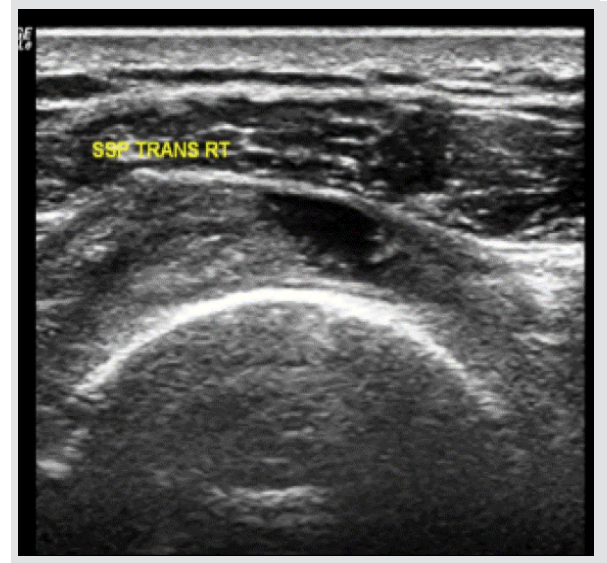

Figure 10: Full thickness tear.

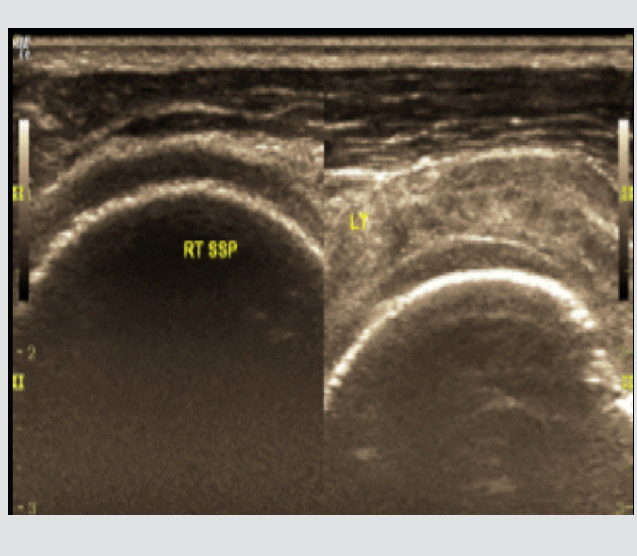

scanning. There may be prominent reflection of the US beam at the interface of fluid and the articular cartilage called as the "cartilage interface sign".

\section{Partial Thickness Tear :}

The main finding of a partial thickness tear on sonography is a focal, localized area of hypoechogenicity, affecting only a part of the tendon thickness. The size of the tear is measured in the short axis in $\mathrm{mm}$ and also as a percentage of the tendon diameter.

Bursal surface tears are seen as focal hypoechoic/anechoic lesions along the bursal surface, but not extending upto the articular surface. Articular surface tears are also seen as focal hypoechoic/anechoic lesions along the articular surface, but not extending upto the bursal surface.

Intrasubstance or interstitial tears can be seen as ill defined focal hypoechoic lesions within the substance of the tendon, not extending either upto the bursal or articular surfaces.

\footnotetext{
17 | Asian Journal of Arthroscopy | Volume 6 | Issue 1 | January-June 2021 | Page 15-23
} 


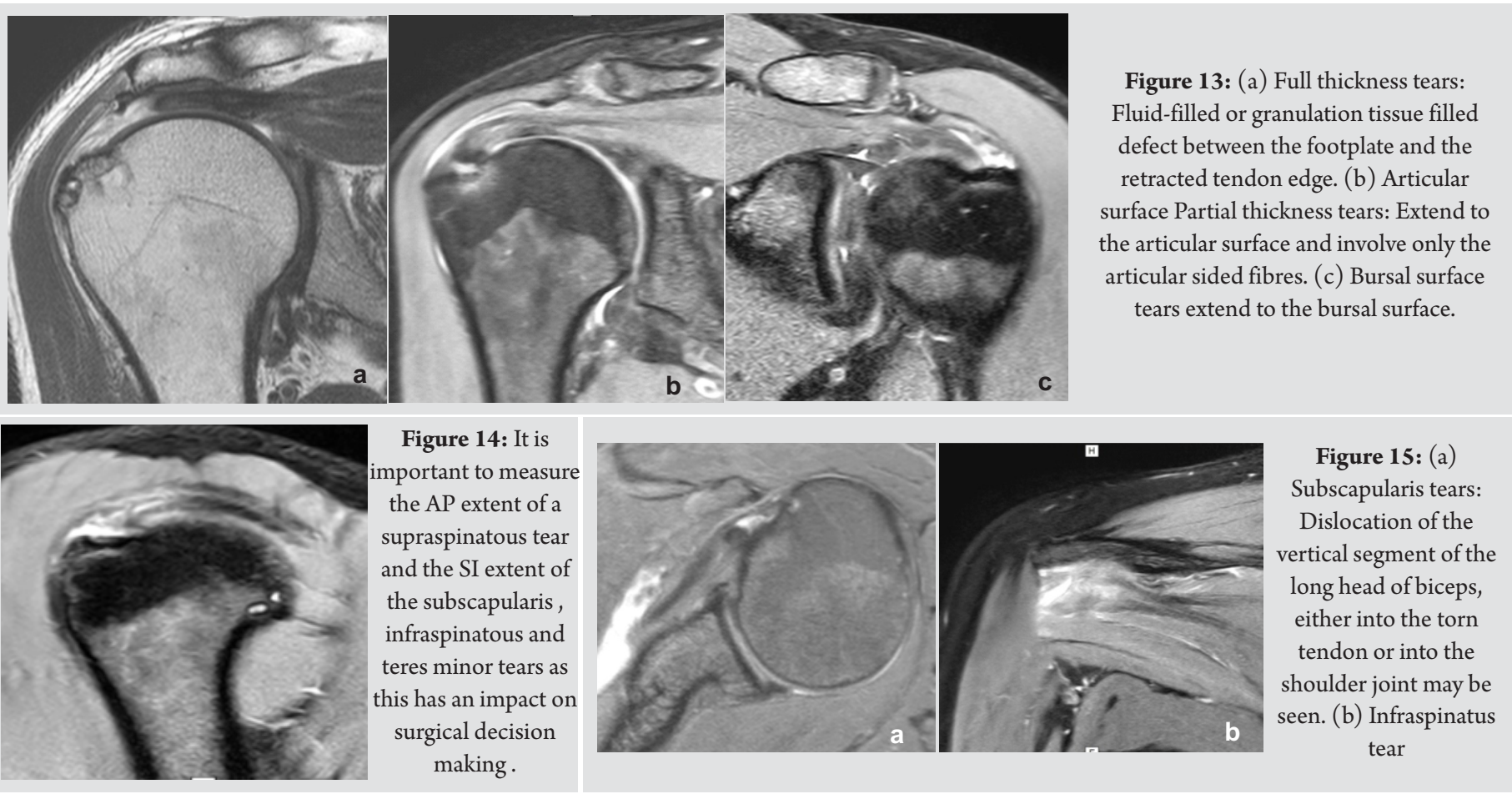

\section{MRI:}

MRI is a better modality of investigation for cuff tears as it also addresses size and degree of fatty infiltration of the muscle belly, diagnoses associated labral tears and evaluates cartilage status .

It is important to look for factors causing external impingement when we see a bursal surface tear.

Interstitial tears do not extend to either the articular or bursal surface. The dimensions of a cuff tear have implications for selection of treatment (conservative vs operative), selection of type of surgery and surgical approach, post operative prognosis and chances of tear recurrence.

The depth/thickness of a partial thickness tear is important to know as increasing depth of tear causes increased strain on the remaining tendon and the rest of the tendons.

\section{Tears may be graded on both USG and MRI into:}

Grade 1 : mild $(<3 \mathrm{~mm})$, grade 2 : moderate $(3-6 \mathrm{~mm})$ and Grade 3 : significant $(>6 \mathrm{~mm})$.

Tears are significant when greater than 50 percent thickness of the tendon is involved and these usually require surgical treatment .

Also, tendon thinning as seen on MRI in the context of a partial thickness tear may be an indication for surgical repair.

\section{(3) Degree of Tendon Retraction}

Tendon retraction is measured as the distance from the footplate till the edge of the torn tendon.

Knowledge of the degree of tendon retraction helps to decide reparability. A tendon is usually not repairable if the retraction is medial to the glenoid fossa.

Unfortunately it is not reliably possible to predict degree of fibrosis in the retracted tendon on USG or MRI.

\section{(4) Shape of the SS Tear}

This is important in deciding the type of repair especially in supraspinatous tears.

\section{(5) Condition of the Involved Tendon}

Degenerated tendon or one that has pre-existing tendinosis is less easy to repair and more likely to undergo re-tear after surgery.

Fibrotic or calcified tendon is more difficult to repair and is likely to cause post operative stiffness and pain. Rotator cuff calcifications can present either as an acute calcific tendonitis or chronic calcific tendinopathy.

\section{Tears can be classified arthroscopically into three basic shapes}

Crescentic tear: tendon pulls away from the greater tuberosity but does not retract far medially and therefore can be re-attached without much tension on the tendon.

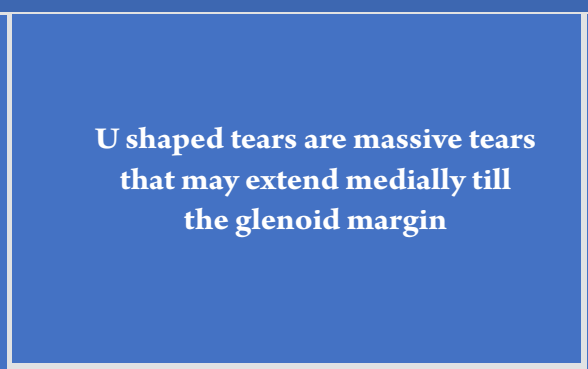

Figure 16: Shapes of supraspinatous tears.
L-shaped tears are massive tears with a longitudinal component along the orientation of the cuff fibres and a transverse component along the cuff insertion 


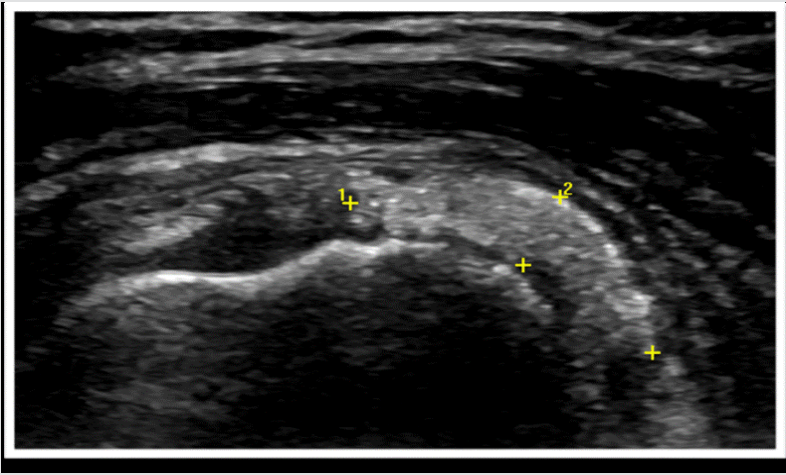

Figure 17: Rotator cuff calcifications appear as intratendinous hyperechoic foci.

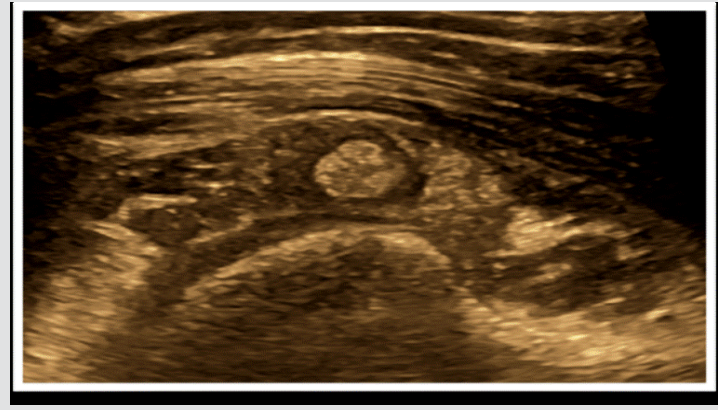

Figure 19: Very rarely calcifications migrate into the muscle bellies and can be seen as ill defined echogenic deposits within the muscles. Therapeutic needling of the tendon calcification can be done using USG guidance
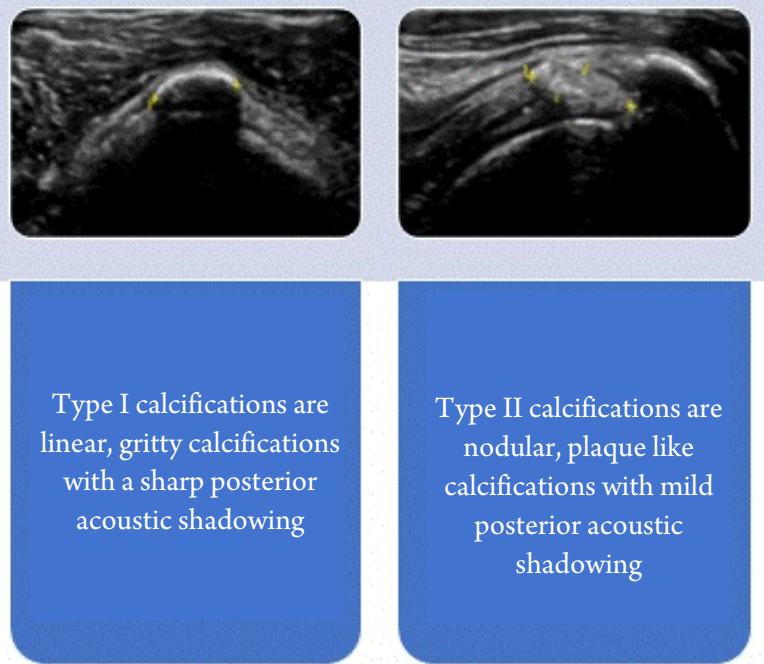

\section{USG :}

Calcifications render the tendon very bulky and demonstrating severe probe tenderness..

\section{MRI:}

Changes of tendinosis will appear iso to mildly hyperintense on the PD images and will not become bright on $\mathrm{T} 2$.

Calcification is better seen on USG or CT scan and may sometimes be missed on MRI. The tendon adjacent to the calcification can

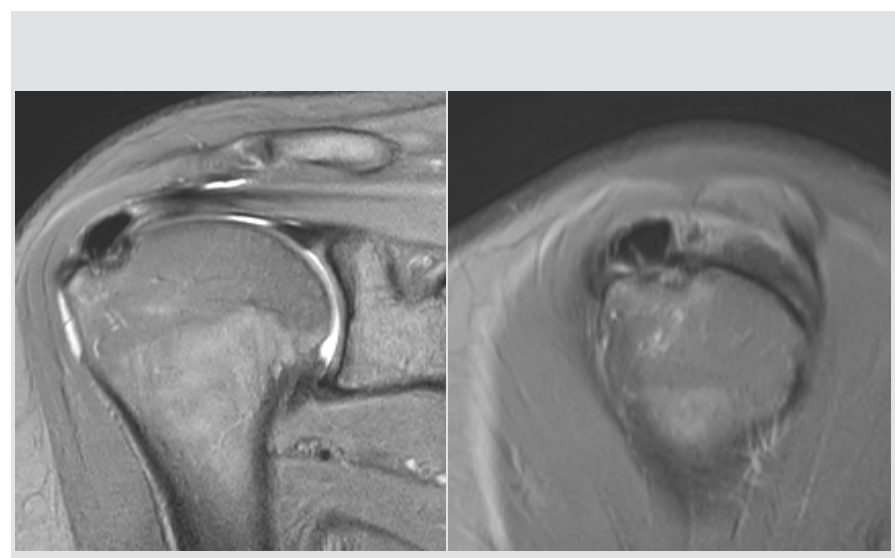

Figure 20: Calcification /ossification appears dark on all pulse sequences and more dark [ blooming] on the gradient $\mathrm{T} 2$ sequences

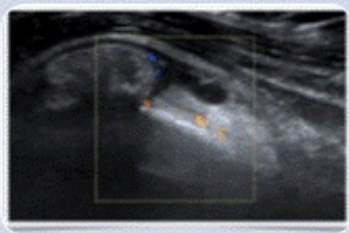

Type III calcifications, are the cheesy, amorphous, paste like calcifications which are ill defined and do not have any posterior acoustic shadowing. The type III calcifications can extrude out into the subacromial bursa resulting in calcific bursitis
Figure 18: Types of tendon calcifications

sometimes tear and calcific deposits get extruded into the subdeltoid bursal space causing inflammation in the bursal space.

\section{(6) Size of Muscle Belly and Fatty Infiltration}

Repair of a cuff tear will not result in optimum outcome if the muscle is atrophic. Both muscle size and degree of fatty infiltration must be evaluated.

NB: Ensure that sagittal oblique images are obtained sufficiently medial to the gleno-humeral joint interface so as to adequately image the muscle belly. As the torn tendon tends to retract, the muscle belly also retracts medially and we may get the false appearance of reduced size of the belly.

Scapular Ratio: If the ratio of the cross sectional area of supraspinatous muscle belly to the cross sectional area of the supraspinatous fossa is less than 50 percent, the muscle is deemed atrophic.

Tangent Sign: A normal sized supraspinatous muscle belly should cross a line drawn between the superior border of the scapular spine and the superior margin of the coracoid process.

Fatty Infiltration: Fatty infiltration in the muscle belly is an important predictive factor for surgical outcome. 


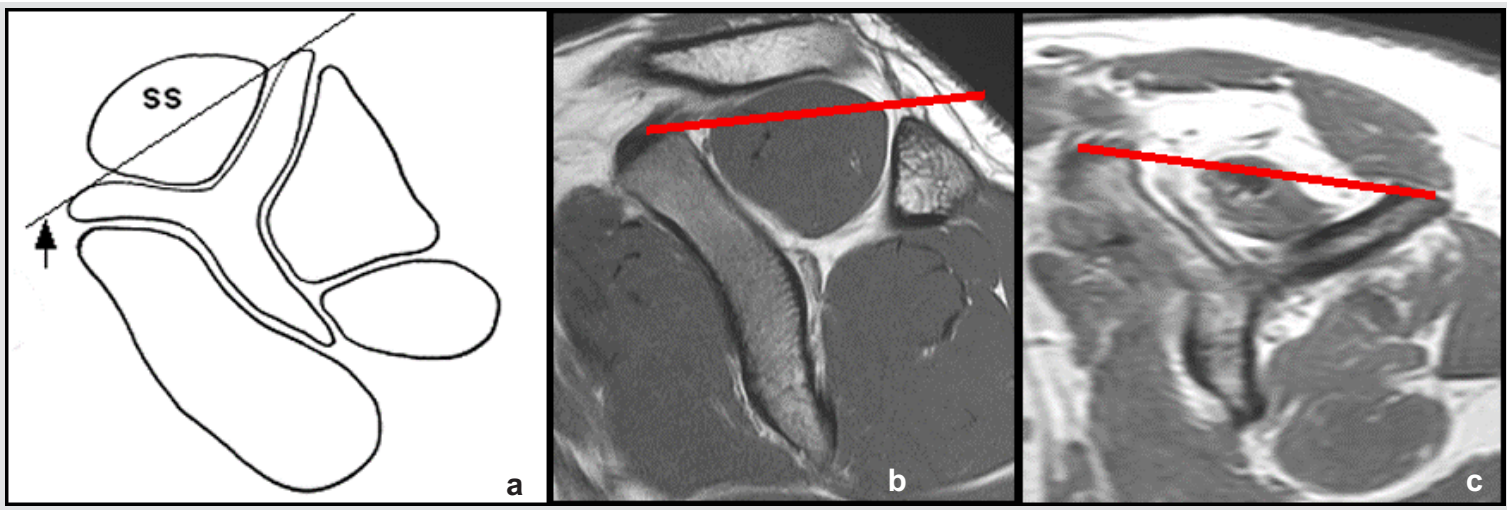

Figure 21: (a, b) Normal supraspinatous muscle bulk (c) Atrophic muscle

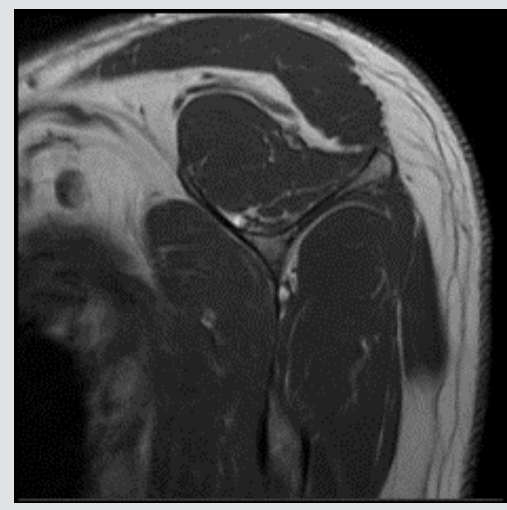

Figure 22: Normal muscle belly. No fatty infiltration seen.

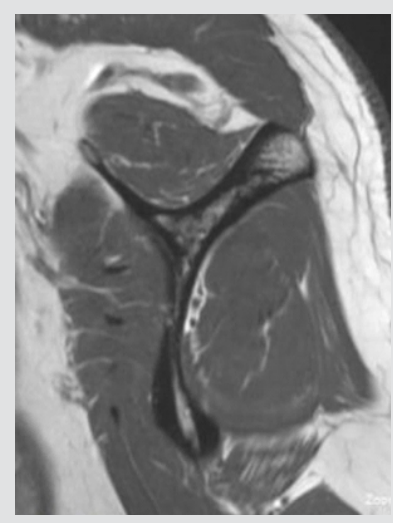

Figure 24: Selective Denervation atrophy of the teres minor muscle belly
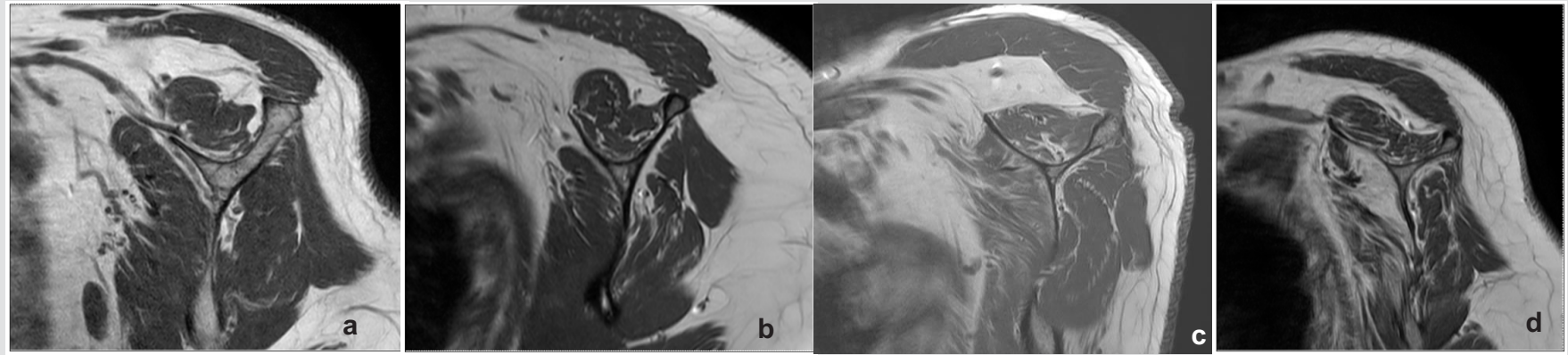

Figure 23: (a) Grade 1- Few areas of fatty infiltration (b) Grade 2 - More areas of fatty infiltration than grade 1, but fat is less in volume than the muscle fibres (c) Grade 3 - Fat infiltration is more than the muscle fibres (d) Grade 4 - Lot of fatty infiltration and very sparse muscle fibres

Goutalliers Classification of Fatty Infiltration in the Muscle Belly:

NB: Fatty infiltration may occur in the teres minor muscle belly in the setting of a cuff tear not involving the teres minor tendon.

\section{(7) Condition of Other Structures: Long Head of Biceps,} Labrum, Rotator Interval

\section{Long Head Biceps :}

Associated degeneration of the long head biceps may cause persistent post operative pain and tenotomy may be required in older patients.

\section{Labrum:}

Posterior cuff tears are associated with posterior superior labral disease in patients with internal impingement.
Anteroinferior labral tears in patients with anterior instability may be associated with supraspinatous tears especially in middle aged women.

Post traumatic anterior labral tears may be associated with subscapularis tears in high intensity trauma.

\section{Rotator Interval Tears}

\section{(8) Factors causing external impingement.}

A) Coraco-acromial arch comprises of anterior $1 / 3^{\text {rd }}$ acromion, coraco-acromial ligament and coracoid process. Anything that causes further narrowing of this already narrow space can cause external impingement. 


\section{Types of Acromion :}

A higher incidence of cuff tears is seen in type 3 and sometimes type 2 likely in association with traction type enthesophyte spurs from the coraco-acromial ligament. Lateral down sloping acromion can also cause external impingement on the supraspinatous tendon, especially if associated with inferior bony spurs.

Thick acromial attachment of the coraco-acromial ligament and presence of osacromiale are associated with cuff impingement.

B) Subcoracoid Impingement: No significant correlation has been found between the width of the subcoracoid space and subscapularis tears.

\section{(9) Cuff Arthropathy}

MRI:

It is important to distinguish osteoarthritis arising from a neglected massive cuff tear from primary osteoarthritis as the surgical treatment is different in the two conditions. MRI helps in making this differentiation.

\section{Role of CT}

1) Trauma : For better evaluation and quantification of fractures $3 \mathrm{D}$ reconstruction is sometimes required.

\section{2) Osteoarthritic shoulder}

\section{(10) Post Operative Imaging \\ MRI:}

Artefacts from surgical anchors/screws etc can impair image clarity in post operative scans. It is however possible to get enough diagnostic information using metal artifact reduction software (MARS protocol) and tweaking the imaging sequences.

MR imaging performed soon after surgery may cause some signal in
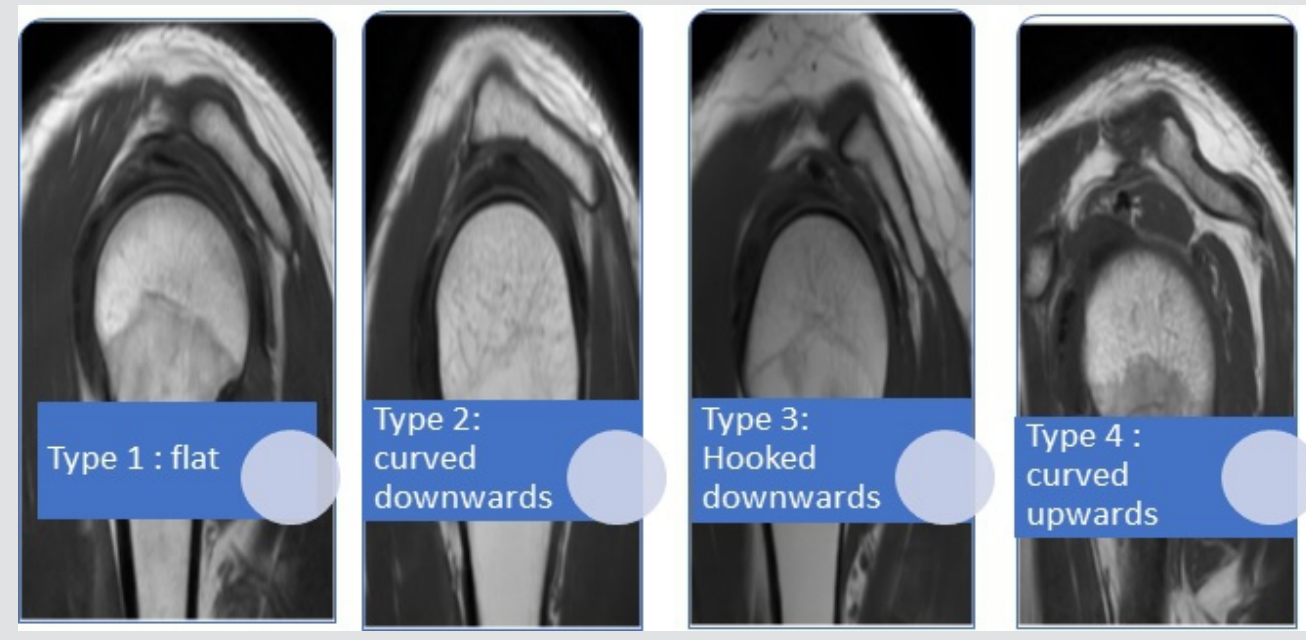

Figure 25: Types of acromion
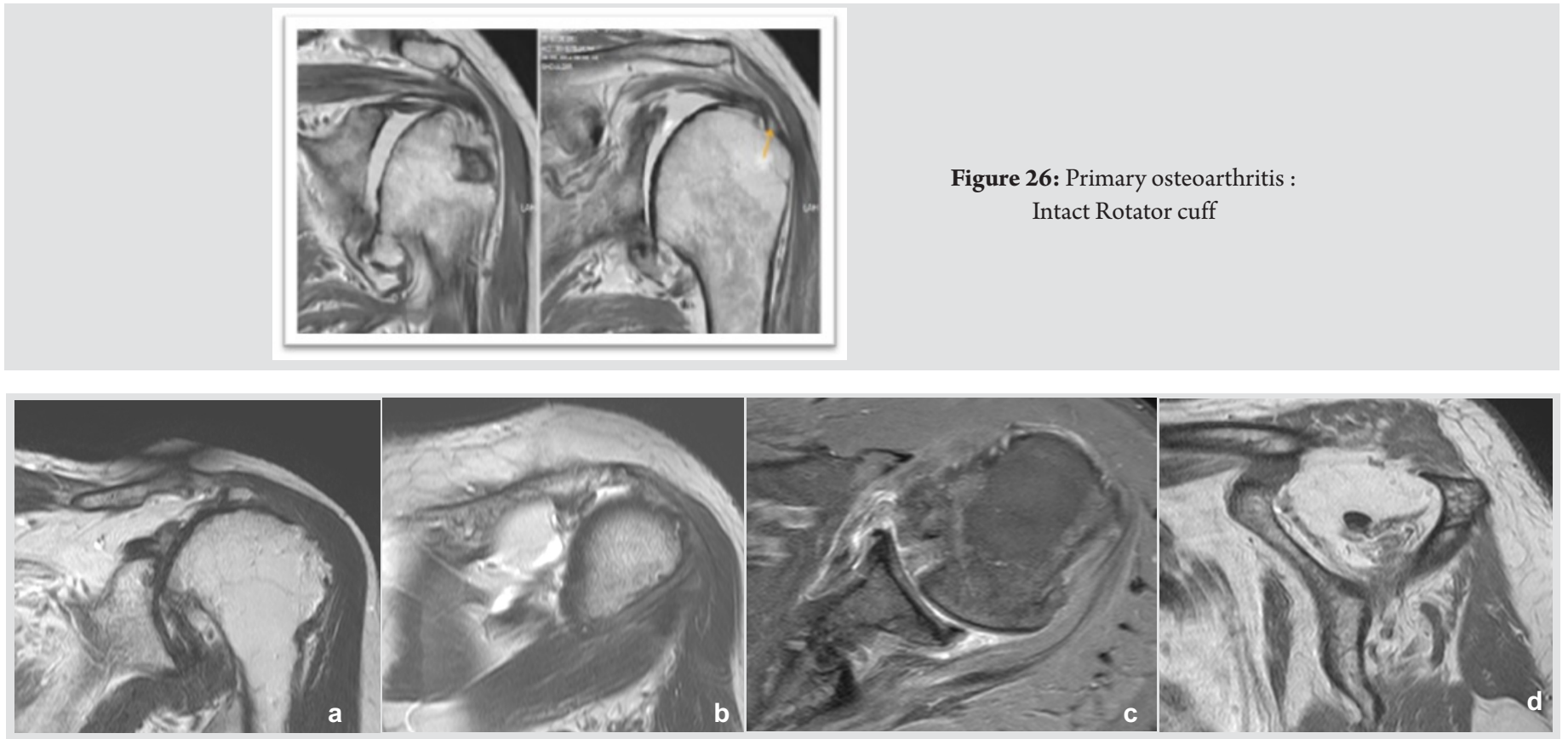

Figure 27: Cuff arthropathy : Neglected and untreated cuff tears can result in osteoarthritic changes in the shoulder 

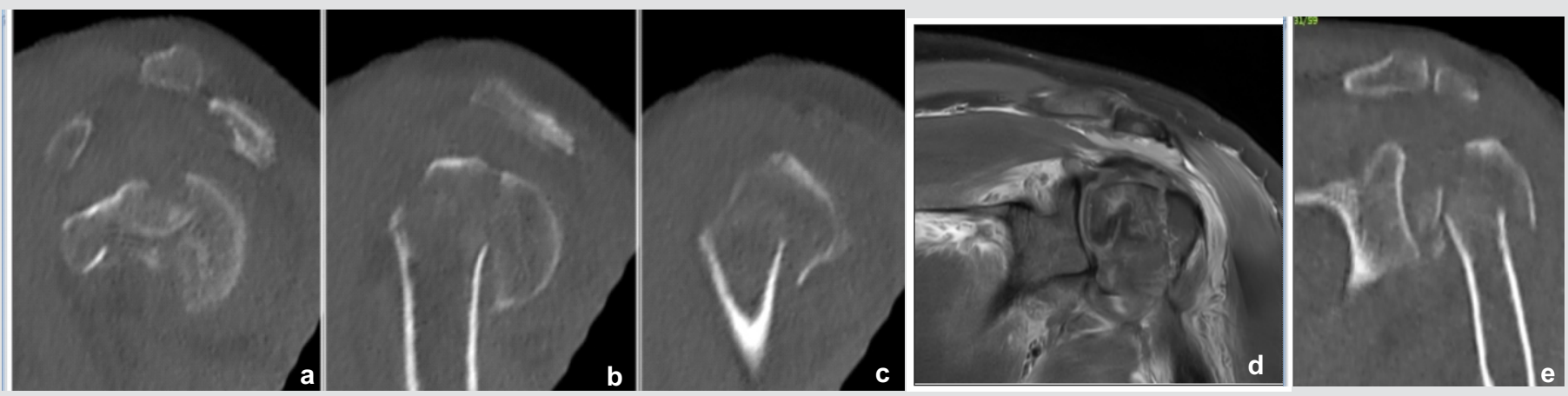

Figure 28: (a-c, e) CT images (d) Coronal PDFS demonstrating fractures

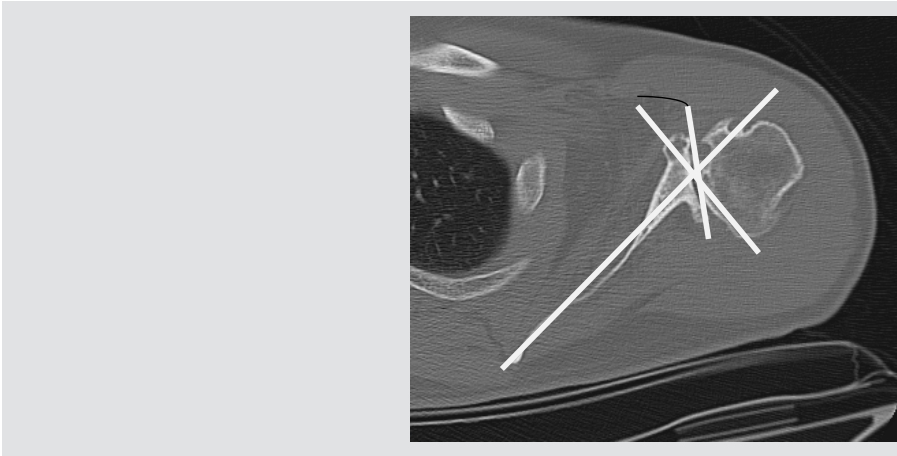

Figure 29: For the calculation of Friedman angle/angle of glenoid retroversion
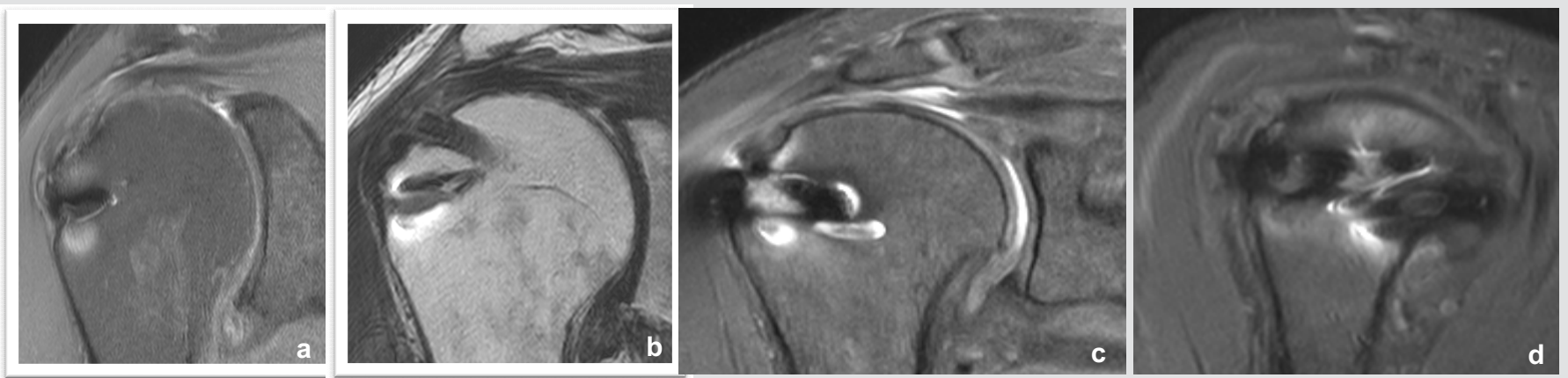

Figure 30: (a) Coronal T2 (b) PD (c, d) PDFS images of a post operative cuff
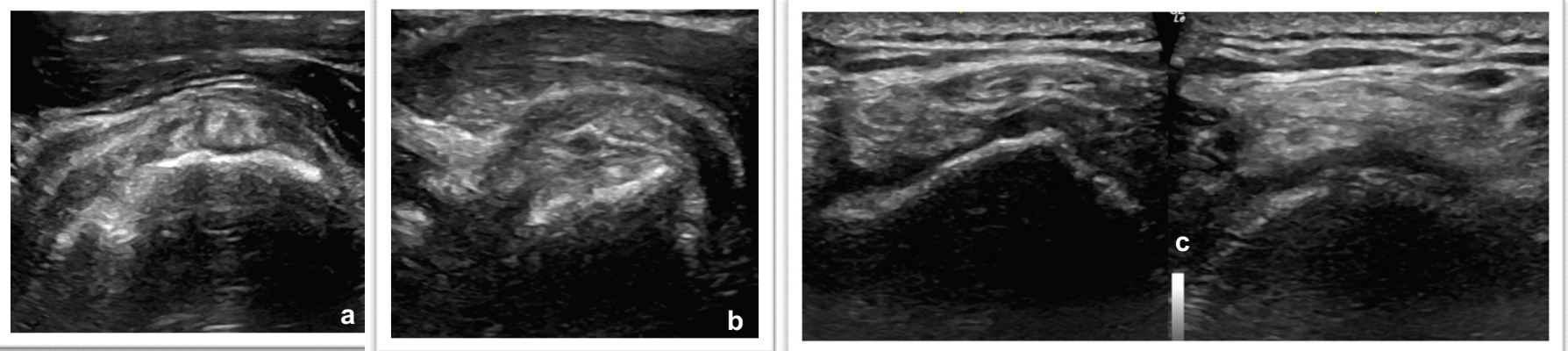

Figure 31: (a) The operated cuff tendons appear more echogenic and heterogenous as compared to the native cuff.

(b) Suture materials appear as linear, thread like material leading upto the suture anchors. Focal defects are often seen within the operated cuff and need not be indicators of retear (c) The most reliable sign of a re tear is nonvisualisation of the cuff tendons and tendon retraction from the surgical trough. 
the intact cuff so it is important to look at $\mathrm{T} 2 \mathrm{~W}$ images .

Re-tear is easily diagnosed as either full thickness avulsion from the footplate or partial thickness tears within the tendon. This will look the same as tears in the non operated cuff.

\section{USG:}

Post operative changes such as troughs, anchors and bio screws can be readily visualized by US. Since no artefacts are created on USG (unlike with MRI/CT), they do not hamper visualization of the rotator cuff tendons.

\section{Therapeutic Use of USG:}

Postoperative stiffness can be addressed by injection of a high volume of fluid (hydrodilatation) underneath the lateral aspect of the coracoid process under USG guidance.

\section{Conclusion}

\section{Diagnostic Imaging should :}

1) Help to identify possible causative factors/inciting factors of cuff tear.

2) Help in the decision making of conservative versus surgical treatment

3) Identify imaging findings that will help in selecting a repair technique.

4) Help to identify those imaging findings that decrease the likelihood of a favourable outcome after cuff repair.

5) Help in the assessment of the post operative cuff.

\section{References}

1. Yamaguchi K, Ditsios K, Middleton WD, Hildebolt CF, Galatz LM, Teefey $S A$. The demographic and morphological features of rotator cuff disease. A comparison of asymptomatic and symptomatic shoulders. J Bone Joint Surg Am.2006;88(8):1699-1704. doi:10.2106/JBJS.E.00835.

2. Moosikasuwan JB, Miller TT, Burke BJ. Rotator cuff tears: clinical, radiographic, and US findings. Radiographics. 2005;25(6):1591-1607. doi:10.1148/rg.256045203.
3. Palmer W, Bancroft L, Bonar F, et al. Glossary of terms for musculoskeletal radiology. Skeletal Radiol. 2020;49(Suppl 1):1-33. doi:10.1007/s00256020-03465-1.

4. Blanchard TK, Bearcroft PW, Constant CR, Griffin DR, Dixon AK. Diagnostic and therapeutic impact of MRI and arthrography in the investigation offull-thickness rotator cufftears. Eur Radiol. 1999;9(4):638642. doi:10.1007/s003300050724.

Declaration of patient consent: The authors certify that they have obtained all appropriate patient consent forms. In the form, the patient has given his consent for his images and other clinical information to be reported in the Journal. The patient understands that his name and initials will not be published, and due efforts will be made to conceal his identity, but anonymity cannot be guaranteed.

\section{Conflict of interest: Nil Source of support: None}

\section{How to Cite this Article}

Patil A, Babhulkar J, Mahadeokar P | Spectrum of Imaging Findings In Rotator Cuff Tears ADescriptive Review | Asian Journal of Arthroscopy | January-June 2021; 6(1): 15-23. 